\title{
Changes in runoff as an indicative measure of water retention status in the Białowieża Primeval Forest
}

\author{
Jan Tyszka 凶, Andrzej Stolarek
}

Forest Research Institute, Department of Forest Ecology, Sękocin Stary, Braci Leśnej 3, 05-090 Raszyn, Poland, phone: 4822 7150526, fax: 22 7150507, e-mail: j.tyszka@ibles.waw.pl

\begin{abstract}
The study concerned changing status of water retention within an unique nature complex of the Białowieża Primeval Forest. The retention was evaluated based on an analysis of research results on water outflow from the lowland catchment of the river Lutownia - representative for physiographic conditions as well as those of habitats and tree stands in Poland's part of the Forest. The catchment in the lowland hydrometric dissection at the Pogorzelce village - Old Białowieża Wilderness, covers the area of $120.1 \mathrm{~km}^{2}$ with natural flow conditions preserved. A unified sequence of measurements carried out since 1966 using unchanged methodology with respect to precipitation, air temperature and forest management status allowed to separate different phases of changes occurring in water resources of this protected forest complex. There were appraised periodical runoff changes and cycles including a decrease in water resources which occurred at the turn of the century. The retention capacity of the catchment was determined based on periodical changes in rainfall-runoff relations and the ongoing process of deteriorating water conditions of forest ecosystem was interpreted.
\end{abstract}

\section{KeY WORDS}

runoff, natural forests, conditions of changes in retention status

\section{INTRODUCTION}

Water resources in the summer 6-month period are crucial for balanced functioning of forest ecosystems. The magnitude of changes in rainfall-runoff relations indirectly indicates catchment retention capacity. During periods of weather oscillations, changes in river outflow volume reflect well the status of water resources, which are generated as a result of the effects of precipitation at the start and then those of evapotranspiration and retention. Rainfall- runoff relations are shaped by specific retention properties of individual forest habitats, which are the reason why discharge changes are not synchronized with the cycle of precipitation incidence. During the period of warmer winters, retention volume is affected not only by changes in precipitation distribution but also by increased rainfall. Long-term identification of discharge of the Lutownia river with its catchment representative for Białowieża Forest conditions allows regular evaluation of the status of retention in the summer 6-month period. Periodical relations between runoff in the summer and winter 6-month periods can be used for forecasting water conditions in the vegetation season. For the past decades, escalating changes in for- 
est water conditions have been reflected in groundwater level decrease and deterioration of river discharge. There is a need for identification of the current state and trends of changes in water resources as well as insightful analyses of the reasons involved in these processes. Unstable hydrothermal conditions manifested as a tendency towards decreasing Sielianinow's index are the main causative factors in the occurrence of disturbances which are threatening nature and proper functioning of forest ecosystems in the Białowieża Primeval Forest (Malzahn and Chomotowska 2009). A substantial role in shaping water resources is played by changing (especially during the periods of weather anomalies) water demands of forest stands, and these depend on forest habitat types (Tyszka 2008). Growing destabilization of water conditions impacts sustainability of the whole valuable and protected forest complex, which is designated as Natura 2000 site 200004 comprising the Białowieża National Park (UNESCO World Heritage Site) and 6 nature reserves "Natural Forests of the Białowieża Primeval Forest". There are also long-established game areas which now are designated among others for the activities connected with reintroduction of the European bison. For conservation of forest ecosystems' natural values and appropriate interpretation of changes in biodiversity, it is crucial to identify territorial water resources of forest habitats, and river runoff is the easiest to evaluate indicative measure of the ongoing changes.

\section{Meterial AND methods}

The aim of the study carried out was to determine changes of water conditions in the forest river catchment with comparatively well preserved natural habitats of the Białowieża Primeval Forest. The issue of water resources is now especially important due to disrupted balance of hydrological relations in this forest complex, exceptional on a world scale. A relatively easy to determine measures of ongoing changes are runoff of catchment and its periodical changeability, and these were used as parameters to assess water resources status in the Białowieża Forest. Runoff of catchment is resultant of the process of shaping other water cycle components, i.e. precipitation, evapotranspiration and retention. The catchment of the Lutownia river, situated in the cen- tral part of the Polish side of the Forest was regarded as representative of its hydrological conditions (Pierzgalski et al. 2002a). Unified and continuous data on precipitation, water levels and discharge were at disposal. These have been collected since 1966 in the natural hydrometric profile preserved by the village Pogorzelce (Old Białowieża Wilderness) following the guidelines elaborated by the Institute of Meteorology and Water Management (IMGW) (Pasławski 1973). Based on average daily water levels as well as the results on volume of water flow under changeable conditions (Byczkowski 1999), there were determined periodical curves of flow intensity and then daily discharges $\left(\mathrm{dcm}^{3} / \mathrm{s}\right)$ were calculated. Uninterrupted registration of changes of other parameters of water cycle constituents was carried out only for three previous years, thus conclusive analyses of the hydrological processes would be too early at that stage.

The amount of catchment average rainfall $(\mathrm{mm})$ was determined by means of polygons of equal rainfall methodology based on the data from four observation stations located in the Forest (fig. 1). Based on daily averages, there were determined rainfall and runoff for the winter 6-month period (November-April) and the summer 6-month period (May-October). There was ascertained the magnitude of periodical changes of half-year and yearly runoff as well as the runoff irregularity coefficient. Taking into account the effect of air temperature there were determined mutual relations between rainfall and runoff in the summer 6-month period (data obtained from IMGW- Białowieża station). The relation between Sielianinow's hydrothermal index and the volume of losses were determined as the difference between the amount of rainfall $(\mathrm{P})$ and runoff $(\mathrm{H})$. Indicative retention capacity of Białowieża Forest stands $\left(\mathrm{R}_{1}\right)$ was determined as the difference between runoff in the summer 6-month period $\left(\mathrm{H}_{\mathrm{L}}\right)$ up to the extreme values of runoff coefficient $\left(\mathrm{c}=\mathrm{H}_{\mathrm{L}} / \mathrm{P}_{\mathrm{L}}\right)$ along with the difference between runoff in winter and summer 6-month periods shaped by winter runoff volume $\left(\mathrm{R}_{2}\right)$. During statistical interpretation (regression equations) of the results, there was taken into account so far gained knowledge on forest and hydrological parameters (Tyszka 2008).

The forest complex analyzed is located on the Polish side of the Białowieża Forest, nearby the border with Belorussia, within the catchments of the river Narewka (right side tributary of the river Narew) and the river Leśna (right side tributary of the river Bug) (fig. 1). The 
sources of both rivers are located beyond Białowieża Forest boundaries, thus the rivers do not reflect specifics of the forest complex investigated. On the other hand, hydrological conditions of the river Łutownia catchment can be presumed as representative (Pierzgalski et al. 2006) for the purpose of this study. The catchment area up to the Pogorzelce cross-section is $120.1 \mathrm{~km}^{2}$, which is $19 \%$ of the area of the Białowieża Forest on the Polish side.

The river Łutownia starts from the Derlicz Peat Bogs, located by the village Nowosady, and its estuary to the river Narew is located inside the Białowieża National Park. Łutownia's tributaries are periodical wa-

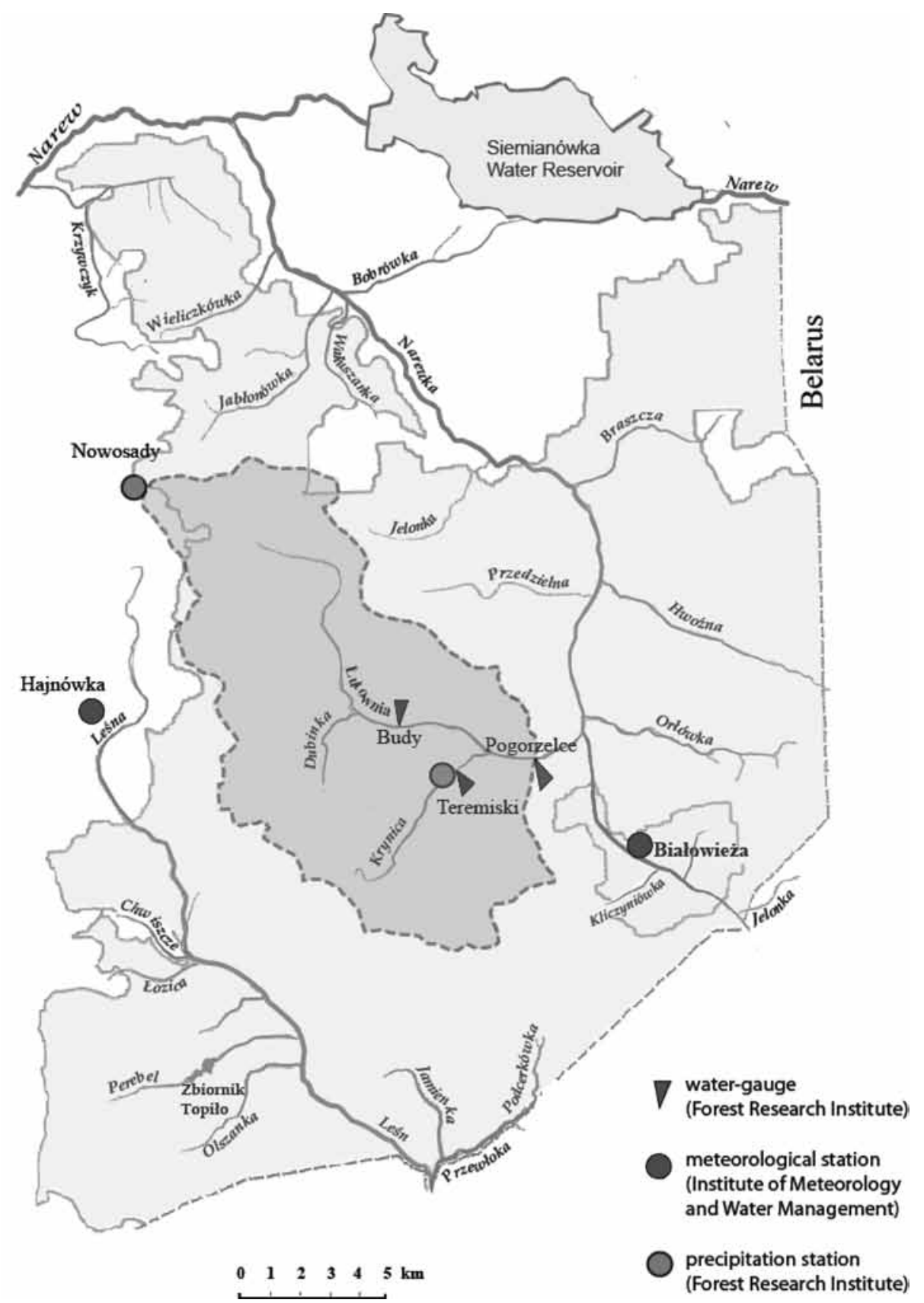

Fig. 1. Position of the Łutownia river catchment within the Polish side of the Białowieża Forest 
tercourses, and only the rivers: Dobitka and Krynica infrequently dry out.

In the 19th century, the Lutownia was regulated for the purpose of its utilization as a water route. In the sixties of the 20th century the river was elutriated. Now it has close to natural character with traces of river bed flattening in the downstream part.

A flat and slightly corrugated plain formed on bottom moraine structures of Middle-Polish glaciations is a prevailing land type within the Lutownia catchment. Glacial tills often reside on a layer of ice-marginal silts of large thickness. The layer of soil was formed on a considerable area from glacial sand and grit, and within marshy river valleys - from low bogs. Average catchment slope is $2.6 \mathrm{~m} / 1 \mathrm{~km}$ at average terrain elevation $166 \mathrm{~m}$ a.s.l. and the height difference $28 \mathrm{~m}$.

Characteristics of habitat and tree stand conditions of the catchment area investigated indicate that almost entire area is covered with a mixed stand with a high production potential. Taking into account generally poor habitats of Middle European Plain terrains, there can be assumed that the area investigated was to our advantage in view of research undertaken due to definite dominance of riparian and alder forest sites over coniferous habitats (9.9\%). Stand species composition shows the highest percentage of spruce $35.6 \%$ followed by oak $-17.5 \%$, pine $-13.4 \%$ and birch $-11.6 \%$. Other admixture deciduous tree species cover $21.9 \%$ of the area. The process of wetland drying out has been ongoing in the area, and in 1983-2010 there was observed a decreasing trend in the groundwater level (Pierzgalski et al. 2002b). During the last decades, overall share of wetland habitats decreased to $28.2 \%$ (counting $16.6 \%$ peat bog habitats) (Majer 2004). Soil moisture changes caused dieback of spruce stands and expansion of the hornbeam. Higher air temperatures, air pollution decrease and limitations of timber acquisition have resulted in a considerable increase of stand stock to more than $300 \mathrm{~m}^{3}$ of large timber wood/ha.

\section{Results}

\section{Water-gauge readings within the hydrometric cross-section Pogorzelce}

As a result of unsupported hydrometric profile, the river Lutownia has unstable conditions of flow, thus its measurements must be conducted often and regularly. Chang- es of flow conditions in the river bed are caused not only by overgrowing vegetation or else ice cover, but also by secondary effects of changes in land elevations as well as river bed slopes. Natural character of the river bed and high fertility of river valley habitats cause abundant overgrowth by deepwater and river bank plants. Water impoundment due to vegetation increases in the period May-August and lasts until the incidence of negative temperatures. Other elements of flow disturbance are connected with the effects of water dames constructed by beavers as well as decayed trees fallen down into the river. Every year, the effects of the above result in periodical water impoundments of tens of centimetres. The flow rate curves obtained for the summer period indicate an increase of the water stages - from $15 \mathrm{~cm}$ to about $25 \mathrm{~cm}$. The maximum elevations are observed in April, and also as transitory incidents - after rainfalls in summer months. Long-term average monthly water levels indicate a tendency to decrease after April snow melting and to increase in the period starting in August and ending in winter. Starting from the year 1974, there was observed a long-term unidirectional change of the river bed level, which raised due to silting by $25 \mathrm{~cm}$ (fig. 2). This was assessed based on river bed probing every time during flow measurements.

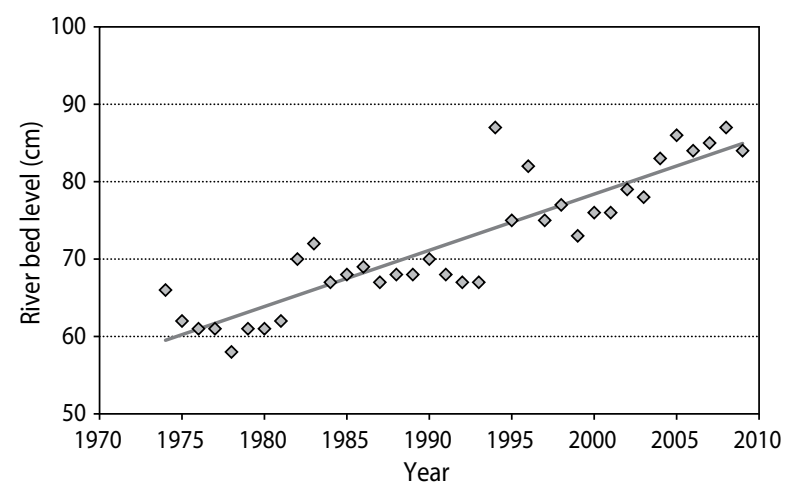

Fig. 2. Shallowing trend in the Łutownia river within the Pogorzelce cross-section

During the annual cycle, the level of the river bed indicates small and short-lived fluctuations. Monthly average changeability of river bed levels registered during the period of observations amounts for a few centimetres, along with the tendency to shallow during the hydrologic year (fig. 3). The lowest river bed level is usually observed in November being a result of increased 
intensity of river erosion owing to dying vegetation. In general low level of the river bed is also observed at the beginning of snow melting period, when ice cover pulls out (February-March) and after the period of river rising (June-August). Silting of the river bed is usually observed during dry months (May-September). In view of that, river bed movements cause a decrease of the amplitude of extreme water levels.

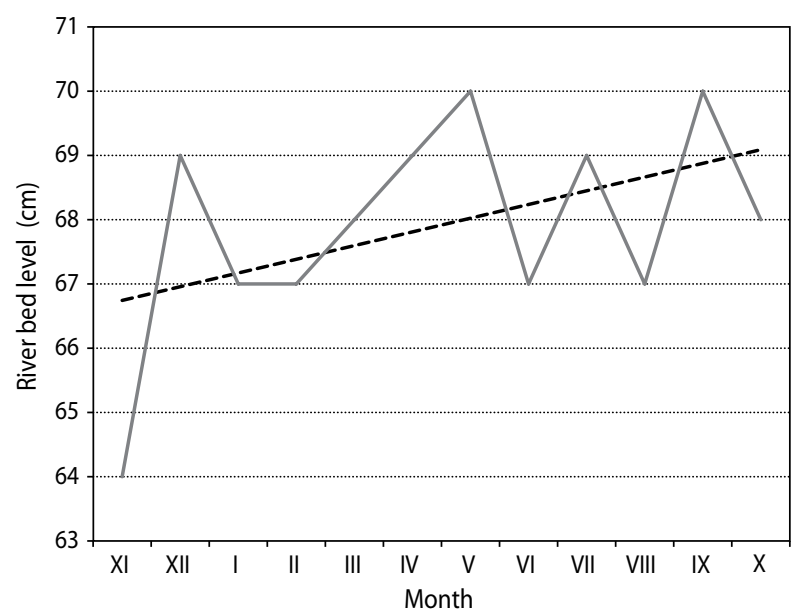

Fig. 3. Average monthly changes of Łutownia's river bed within the cross-section Pogorzelce

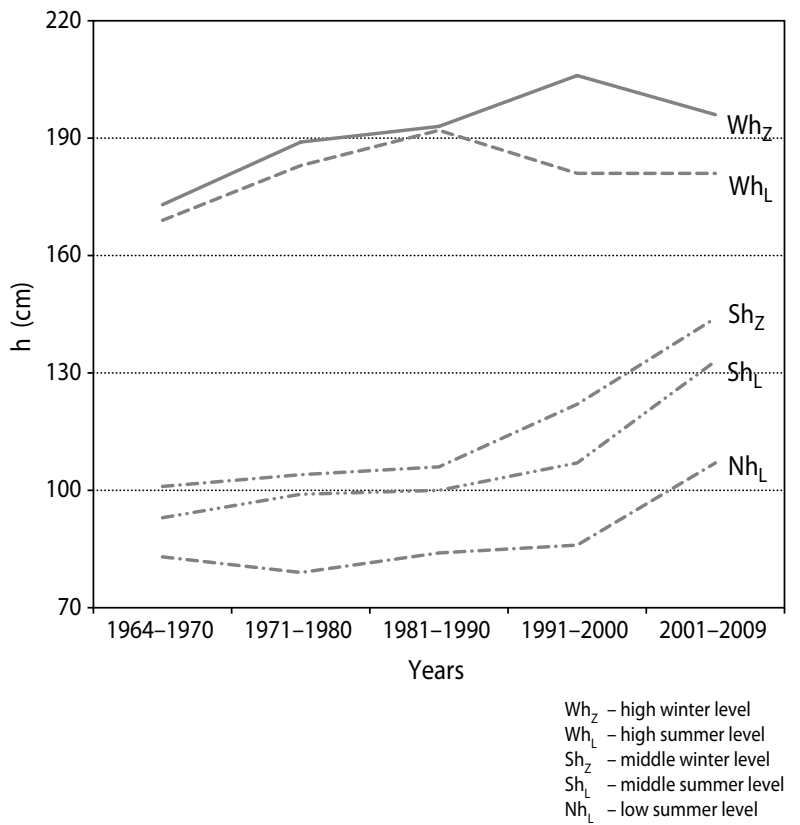

Fig. 4. Process of decade changes in typical water levels in the Łutownia river
Even though river flow rate decreases in the Pogorzelce hydrometric cross-section, disturbances of water outflow in the river bed (which started in the year 1990) caused the increase of low and middle river water levels. High water levels were unchanged during the summer 6-month period and indicated an increasing trend during the winter 6-month period (fig. 4). As a result, a decrease of the runoff rate did not lead to drying out of habitats along Lutownia's lower stream (1.4 km section) flowing inside the Białowieża National Park.

Ruling out any activities on river stream restoration within the areas under strict protection of nature resources obstructs conducting hydrometric measurements. However, thank to the implementation of proven methods of interpretation of runoff and comparisons of the results obtained with those reported on runoff of the catchment in the area investigated as well on subcatchments of the Łutownia river (fig. 1), there can be assumed that our results were affected only by the minor error. On a regular basis, river discharge volume is the constituent of the water balance which can be assessed most exactly, even under the conditions of natural hydrometric cross-sections. This is possible thank to recent development of technical methods of flow measurements.

\section{Long-term changes in river discharge}

Several studies have been conducted on discharge within forest catchments in north-eastern Poland (Kucharska et al. 1984; Olszewski 1986; Ciepielowski et al. 1992; Byczkowski and Mandes 1998; Tyszka 2007). The results of these works confirmed the specific character of runoff and the unquestioned role of forest areas in the water cycle. Based on the example of the Łutownia river we analyzed the volume and timing of runoff from the forest of a primeval type. Evaluation of the tendencies of changes ongoing in retention resources of the catchment was carried out with the use of long-term results (since 1959) on precipitation and discharge. Hydrological data from the period 1959-1962, i.e. just before elutriation of the river bed, indicated that at annual rainfall lower than normal by $31.5 \mathrm{~mm}$, overall runoff stayed at the similar level in the following years. Advantageous retention capacity of the catchment under the conditions of not disturbed water cycle was confirmed by higher (more than a dozen \%) runoff observed in the 
summer 6-month period as well as that in the winter 6-month period - in the same way lower. After 1966, runoff relied upon overall increase of air temperature and weather anomalies (Pierzgalski and Tyszka 2005). There is a possibility that after the year 2005, small retention objects introduced in the catchment area affected Łutownia's discharge.
After the year 1983, there was observed a growing rainfall deficiency during the summer 6 -month period which resulted in decreased runoff. Until the year 2010, balance losses in the Lutownia catchment increased by approximately $3.1 \mathrm{~mm} /$ year. This resulted in lowering groundwater level with an extent depending on habitat wetness (Pierzgalski et al. 2002b). Average hydrother-

Tab. 1. Average hydrothermal parameters of the Lutownia river catchment in 1966-2011

\begin{tabular}{|c|c|c|c|c|c|c|c|c|}
\hline & Reriod & $\begin{array}{c}\text { Rainfall } \\
\mathrm{P}(\mathrm{mm})\end{array}$ & $\begin{array}{c}\text { Runoff } \\
\mathrm{H}(\mathrm{mm})\end{array}$ & $\begin{array}{c}\mathrm{P}-\mathrm{H} \\
(\mathrm{mm})\end{array}$ & $\mathrm{c}=\mathrm{H} / \mathrm{P}$ & $\begin{array}{c}\text { Air } \\
\text { temperature } \\
\mathrm{T}\left({ }^{\circ} \mathrm{C}\right)\end{array}$ & $\begin{array}{c}\text { Sielianinow's } \\
\text { index } \\
\mathrm{k}_{\mathrm{L}}=\mathrm{P} / 0,1 \mathrm{nt}\end{array}$ & $\begin{array}{c}\text { Duration (days) } \\
\text { period }\end{array}$ \\
\hline Nov-Apr & 235.2 & 69.0 & 166.2 & 0.297 & -0.2 & & & $29.12-27.02$ \\
\hline May-Oct & 395.2 & 37.4 & 357.9 & 0.093 & 13.7 & 1.58 & $7.05-24.09$ & \\
\hline Nov-Oct & 630.4 & 106.4 & 524.1 & 0.169 & 6.8 & & & \\
\hline
\end{tabular}

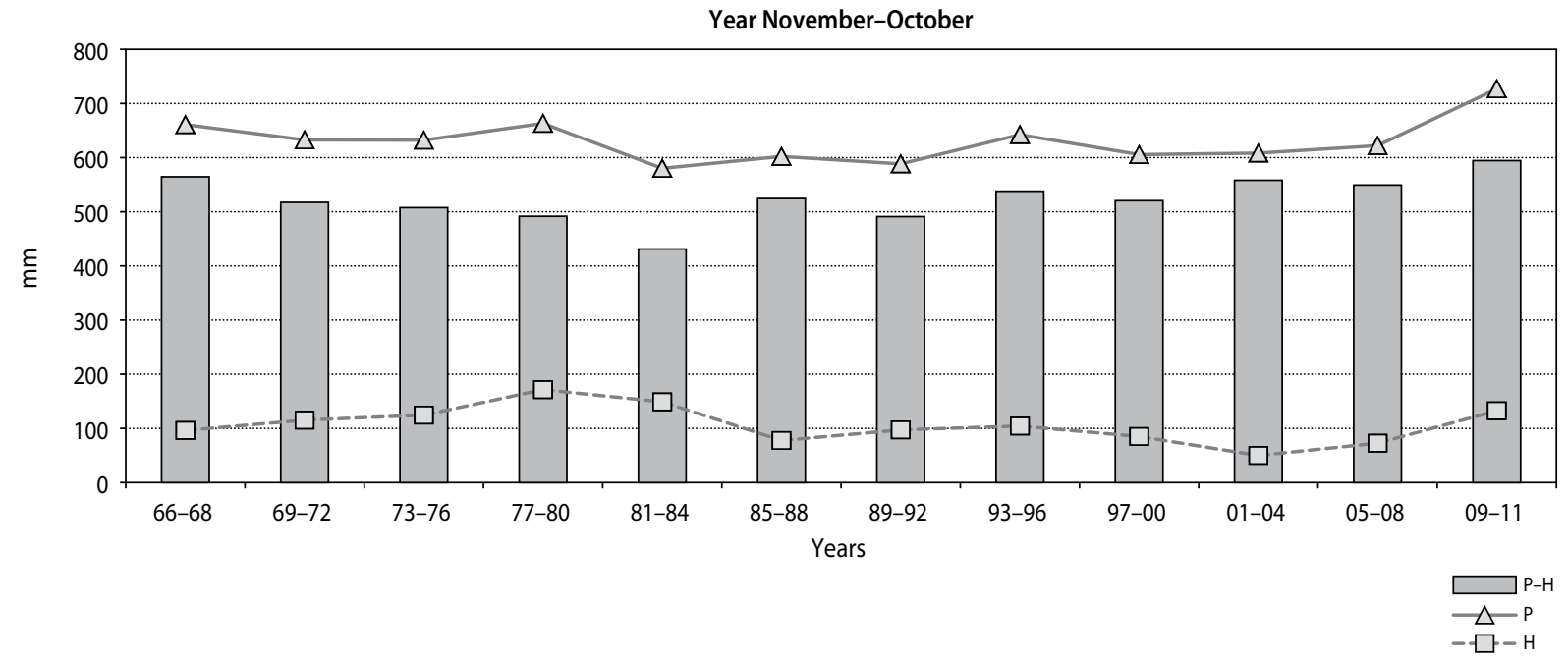

Summer 6-month period May-October

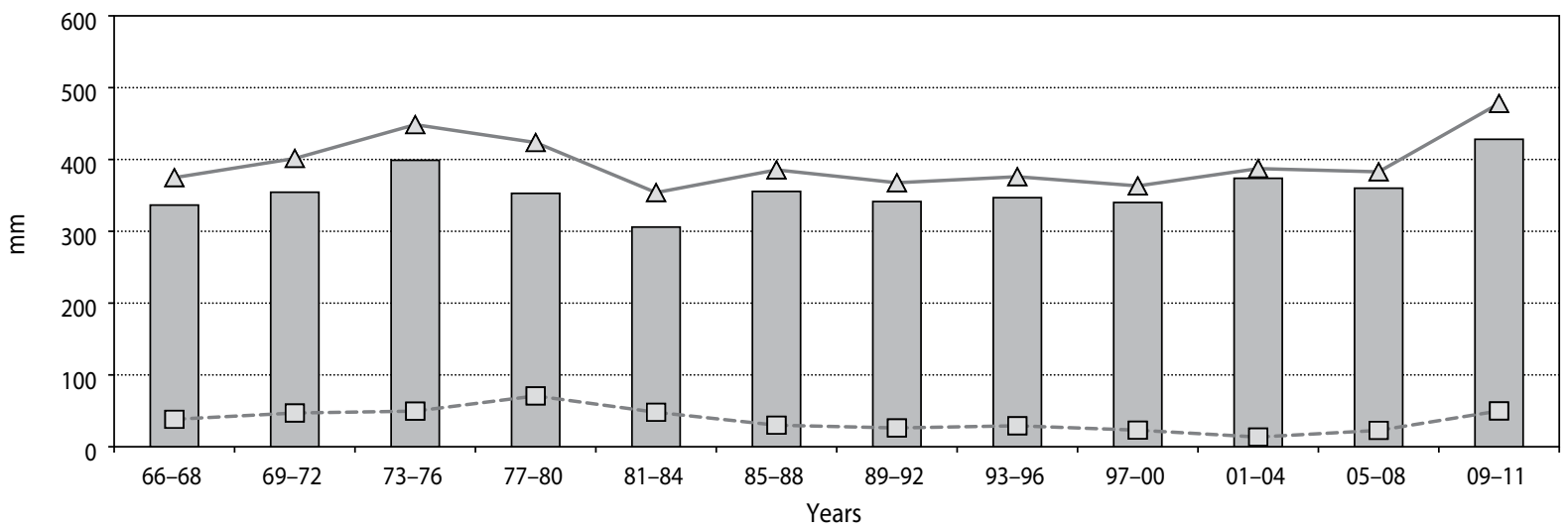

Fig. 5. Average values of rainfall $(\mathrm{P})$, runoff $(\mathrm{H})$ and balance losses $(\mathrm{P}-\mathrm{H})$ within the Łutownia river catchment in 4-year periods 


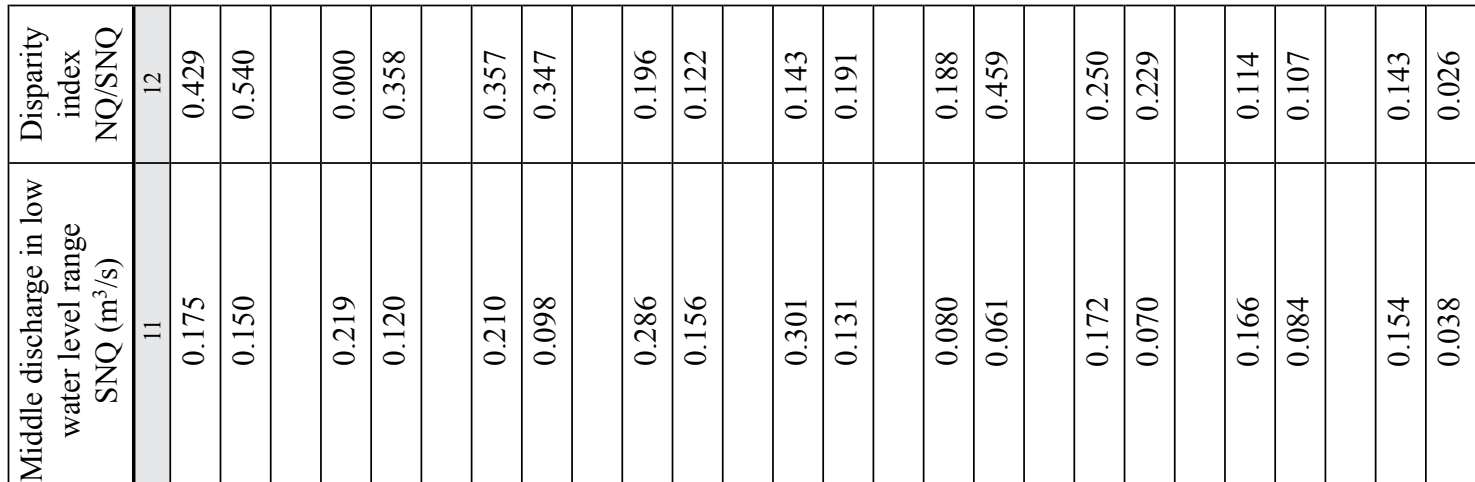

\begin{tabular}{|c|c|c|c|c|c|c|c|c|c|c|c|c|c|c|c|c|c|c|}
\hline 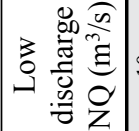 & $\circ \stackrel{n}{a}$ & $b_{0}^{c}$ & $\begin{array}{l}8 \\
0 \\
0\end{array}$ & 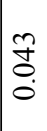 & & 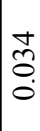 & $\begin{array}{l}0 \\
ٌ \\
0 \\
0\end{array}$ & $\begin{array}{l}\stackrel{\partial}{0} \\
\dot{0}\end{array}$ & 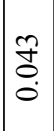 & $\stackrel{2}{2} 0$ & $\begin{array}{l}n \\
0 \\
0\end{array}$ & 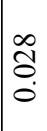 & 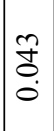 & $\begin{array}{l}0 \\
0 \\
0 \\
0\end{array}$ & $\frac{a}{0}$ & $\begin{array}{l}\stackrel{\text { oे }}{0} \\
\text { o. }\end{array}$ & ָ̃ & $\begin{array}{l}\overrightarrow{0} \\
0 \\
0\end{array}$ \\
\hline
\end{tabular}

응

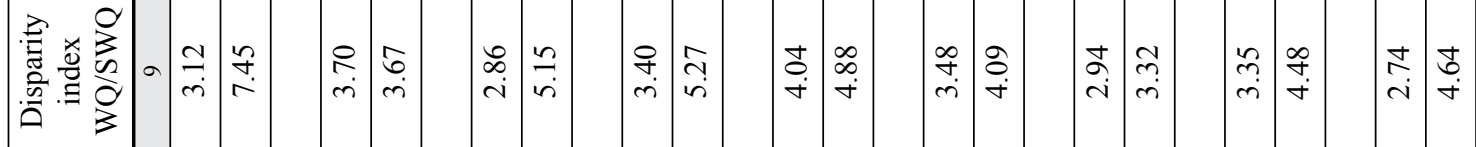

.

跑

o.

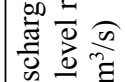

:

กิ

굴

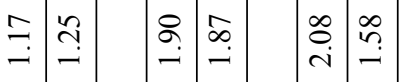

ळे

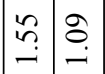

$\stackrel{n}{\rightarrow}$

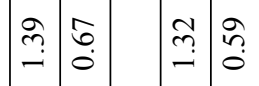

.

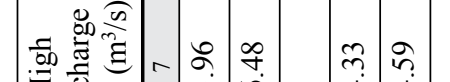

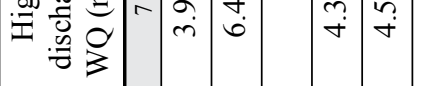

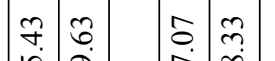

莳

în.

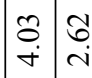

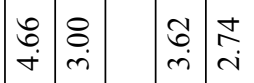

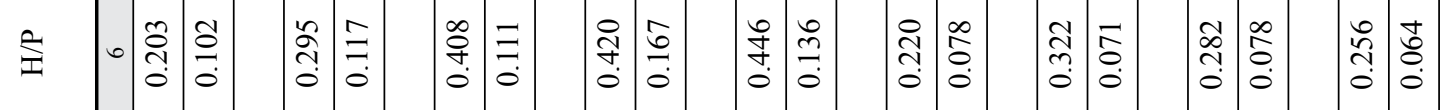

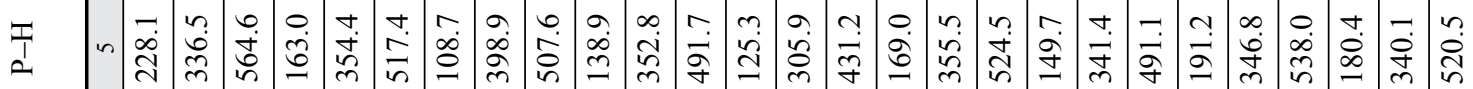

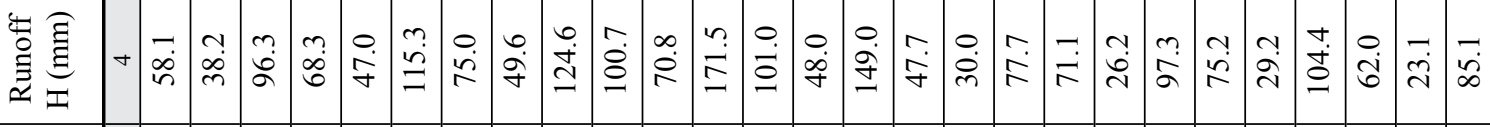

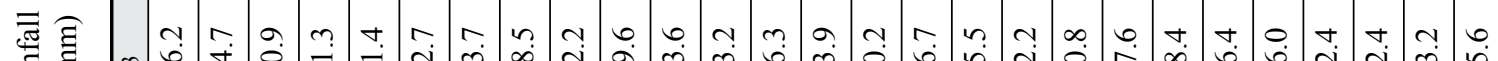

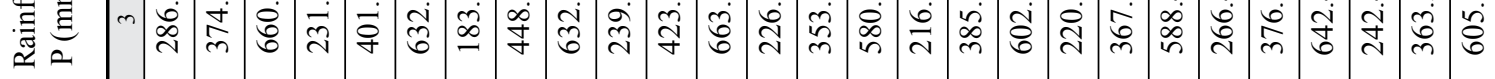

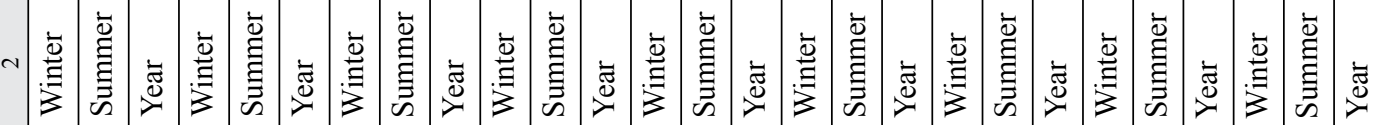

$\sum_{\bar{\Xi}}^{\infty}$

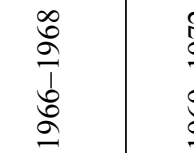

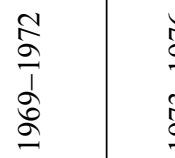

$\stackrel{2}{a}$
$\stackrel{1}{2}$
$\stackrel{2}{a}$

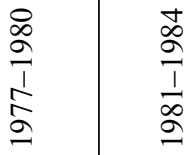

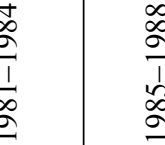

$\infty$
$\infty$
$\infty$
$\infty$
$\infty$

๙ั

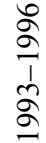

\&

ลे 


\begin{tabular}{|c|c|c|c|c|c|c|c|c|c|c|c|c|c|c|}
\hline$\simeq$ & $\begin{array}{l}8 \\
8 \\
\vdots \\
0\end{array}$ & $\mid \begin{array}{l}0 \\
0 \\
0 \\
0\end{array}$ & & $\begin{array}{l}0 \\
\tilde{n} \\
\tilde{n}\end{array}$ & $\begin{array}{l}\grave{\vdots} \\
0 \\
0\end{array}$ & & $\left|\begin{array}{l}\infty \\
\infty \\
\infty \\
0 \\
0\end{array}\right|$ & $\overline{\overrightarrow{\hat{T}}}$ & $\begin{array}{l}0 \\
\tilde{3} \\
0\end{array}$ & 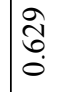 & & $\mid \begin{array}{l}8 \\
\vdots \\
0 \\
0\end{array}$ & $\begin{array}{l}8 \\
\vdots \\
0\end{array}$ & \\
\hline$=$ & ô & $\mid \begin{array}{l}0 \\
\tilde{O} \\
0 \\
0\end{array}$ & & $\stackrel{m}{\stackrel{m}{0}}$ & $\begin{array}{l}\widetilde{Z} \\
\stackrel{0}{0} \\
\dot{0}\end{array}$ & & $\mid$\begin{tabular}{c|}
$\vec{\sigma}$ \\
$\stackrel{\sim}{0}$
\end{tabular} & $\begin{array}{l}0 \\
0 \\
0 \\
0\end{array}$ & $\begin{array}{l}\delta \\
\tilde{n} \\
0\end{array}$ & $\frac{0}{2}$ & & $\left|\begin{array}{l}0 \\
0 \\
0 \\
0\end{array}\right|$ & 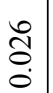 & \\
\hline$\stackrel{\circ}{\circ}$ & $\begin{array}{l}\infty \\
\tilde{e} \\
0 \\
0\end{array}$ & $\mid \begin{array}{l}0 \\
\vdots \\
0 \\
0\end{array}$ & & $\mid \begin{array}{l}R \\
0 \\
0 \\
0\end{array}$ & हे & & $\left|\begin{array}{l}\infty \\
0 \\
0 \\
0\end{array}\right|$ & $\stackrel{2}{0}$ & $\begin{array}{l}\infty \\
0 \\
0 \\
0\end{array}$ & $\begin{array}{l}\vec{\infty} \\
0 \\
0\end{array}$ & & $\mid \begin{array}{l}8 \\
\vdots \\
0\end{array}$ & $\begin{array}{l}8 \\
\vdots \\
\circ\end{array}$ & \\
\hline a & $\begin{array}{l}\tilde{2} \\
\tilde{n} \\
\tilde{m}\end{array}$ & $\begin{array}{l}8 \\
\\
\end{array}$ & & ঙे & $\begin{array}{l}\infty \\
\infty \\
+\end{array}$ & & $=$ & $\frac{0}{1}$ & $=$ & 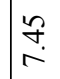 & & 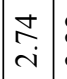 & $\stackrel{\sim}{m}$ & \\
\hline$\infty$ & $\hat{\sigma}$ & $\mid$\begin{tabular}{l}
0 \\
\hdashline \\
0 \\
0
\end{tabular} & & గn & ?ี & & $\left|\begin{array}{l}0 \\
0 \\
0\end{array}\right|$ & $\begin{array}{l}\infty \\
? \\
0\end{array}$ & $\stackrel{\infty}{\stackrel{\infty}{i}}$ & 冓 & & กิ & กै? & \\
\hline In & $\begin{array}{l}2 \\
\dot{r} \\
\dot{r}\end{array}$ & $\begin{array}{c}0 \\
i \\
i\end{array}$ & & $\stackrel{\overbrace{}}{\sim}$ & $\stackrel{?}{?}$ & & $\begin{array}{l}\tilde{N} \\
\tilde{n}\end{array}$ & $\stackrel{i}{i}$ & $\stackrel{t}{i}$ & $\hat{b}$ & & 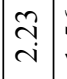 & $\stackrel{?}{n}$ & \\
\hline 0 & $\begin{array}{l}0 \\
0 \\
\ddot{0}\end{array}$ & $\mid \begin{array}{l}n \\
\tilde{o} \\
0\end{array}$ & & 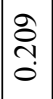 & $\begin{array}{l}8 \\
0 \\
0\end{array}$ & & $\begin{array}{c}n \\
\tilde{n} \\
\tilde{c} \\
0\end{array}$ & $\frac{1}{0}$ & 芩. & $\frac{5}{0}$ & & $\mid \begin{array}{c}0 \\
0 \\
0 \\
0\end{array}$ & $\begin{array}{l}\tilde{n} \\
\tilde{o} \\
\dot{0}\end{array}$ & \\
\hline in & $\begin{array}{l}\stackrel{+}{+} \\
\stackrel{\infty}{-}\end{array}$ & $\begin{array}{l}\infty \\
\stackrel{\infty}{\infty} \\
\stackrel{m}{n}\end{array}$ & $\begin{array}{l}n \\
\infty \\
n \\
n\end{array}$ & $\begin{array}{l}\ddot{2} \\
\stackrel{\infty}{\infty}\end{array}$ & $\begin{array}{l}0 \\
\dot{8} \\
i \\
n\end{array}$ & $\begin{array}{l}\vec{a} \\
\dot{a} \\
\dot{n}\end{array}$ & $\mid \begin{array}{l}\vec{t} \\
\dot{0} \\
0 \\
-\end{array}$ & $\mid \begin{array}{c}\vec{\infty} \\
\stackrel{\alpha}{f} \\
f\end{array}$ & 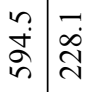 & $\begin{array}{l}\vec{\infty} \\
\mathcal{F}\end{array}$ & $\begin{array}{l}n \\
\dot{J} \\
\tilde{n}\end{array}$ & $\begin{array}{l}\stackrel{r}{\infty} \\
\stackrel{0}{0}\end{array}$ & ڤેे & $\frac{\stackrel{y}{g}}{\mathscr{F}}$ \\
\hline 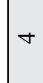 & $\begin{array}{l}0 \\
\dot{0} \\
\dot{n}\end{array}$ & $\begin{array}{l}\because \\
\check{2}\end{array}$ & $\vec{i}$ & $\vec{a}$ & $\begin{array}{l}\infty \\
\grave{d}\end{array}$ & $\stackrel{i}{i}$ & \begin{tabular}{c|}
0 \\
$\dot{D}$ \\
$\infty$
\end{tabular} & $\begin{array}{l}\sigma \\
\dot{q}\end{array}$ & $\begin{array}{ll}\tilde{n} & 0 \\
\tilde{n} & 0 \\
\end{array}$ & $\stackrel{\infty}{\stackrel{0}{R}}$ & $\frac{n}{\Sigma}$ & $\mid \begin{array}{l}0 \\
\dot{0} \\
\text { r. }\end{array}$ & $\ddot{n}$ & $\overrightarrow{n^{\prime}}$ \\
\hline$m$ & $\begin{array}{l}0 \\
\dot{\vec{\lambda}} \\
\text { ते }\end{array}$ & $\mid \begin{array}{l}m \\
\stackrel{n}{\infty} \\
\infty \\
m\end{array}$ & $\begin{array}{l}m \\
\infty \\
0 \\
0\end{array}$ & ڤે & $\begin{array}{c}\infty \\
\mathbb{I} \\
\infty \\
ల\end{array}$ & $\overrightarrow{\tilde{i}}$ & $\begin{array}{c}\stackrel{0}{a} \\
\stackrel{+}{\sim}\end{array}$ & \begin{tabular}{|l|}
$\stackrel{0}{0}$ \\
$\stackrel{\infty}{0}$ \\
$\stackrel{\gamma}{\sigma}$
\end{tabular} & 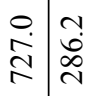 & $\begin{array}{l}0 \\
\infty \\
\stackrel{\infty}{f} \\
\stackrel{9}{*}\end{array}$ & 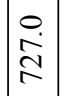 & $\begin{array}{l}\vec{\infty} \\
\dot{\infty} \\
-\end{array}$ & $\begin{array}{l}\hat{n} \\
\hat{n}\end{array}$ & $\begin{array}{l}n \\
\infty \\
\infty \\
n\end{array}$ \\
\hline N & 樆 & 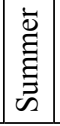 & $\underset{\nabla}{\approx}$ & 离 & 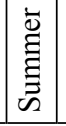 & $\begin{array}{c}\ddot{\vec{J}} \\
\stackrel{\nu}{*}\end{array}$ & 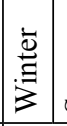 & : & 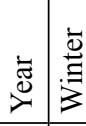 & 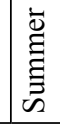 & 离 & 离 & 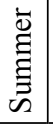 & 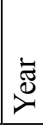 \\
\hline & & 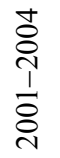 & & & 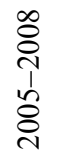 & & & 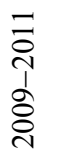 & & $\sum_{\Sigma}^{\star x}$ & & & $\dot{\Sigma}$ & \\
\hline
\end{tabular}

mal parameters with reference to the period of observation are presented in tab. 1.

Climatic conditions of the Białowieża Primeval Forest indicate characteristics of cool and dry continental climate with average annual temperature $6.8^{\circ} \mathrm{C}$ and average summer 6-month period temperature $13.7^{\circ} \mathrm{C}$ (data from Białowieża meteorological station). Average annual rainfall (P) for 46 years of observation was $630.4 \mathrm{~mm}$, and showed advantageous distribution with $63 \%$ rainfall in the summer 6 -month period. Runoff $(\mathrm{H})$ amounted to $106.4 \mathrm{~mm}$, and runoff irregularity coefficient $\mathrm{c}=\mathrm{H} / \mathrm{P}$ was 0.169 . Average balance loss $\mathrm{P}-\mathrm{H}$ per year was $524 \mathrm{~mm}$ being higher than average precipitation in dry years.

Long-term observations on runoff allowed identification of certain regularities in the process of changes. Hydrologic characteristics of the catchment in subsequent 4-year periods of cyclical changes are presented in tab. 2 .

In the last decade of observations, there was observed the increase of discharge irregularity coefficient in the high range of water levels WQ/SWQ as well as discharge in the low range NQ/SNQ. Fig. 5 shows an increasing tendency of the difference between rainfall and runoff since 1981, both in the summer and winter 6-month periods. The results indicate great periodical changes in the process of shaping water resources status.

Reducing effects of climatic and hydrologic drought is crucial for stable development of wetland habitats. The analysis of occurrence of periodical low rainfall in the summer 6-month periods indicates that low rainfall showed once in 3-6 years and its volume was smaller by $21 \%$ when compared to normal rainfall. Except for the year 2000, there was observed a general increase of minimal rainfall in the summer 6-month period (fig. 6).

Opposite tendencies were indicated in the case of minimal runoff in the summer 6-month period (fig. 7). Even though minimal rainfall was relatively high, decreasing trends were observed for low (NQ) and middle (SNQ) discharges in a range of low water levels until the year 2005. The lowest values of discharge in 4-year periods were observed in the turn of the century, when water discharge from the Lutownia river almost vanished around the year 2000 . 


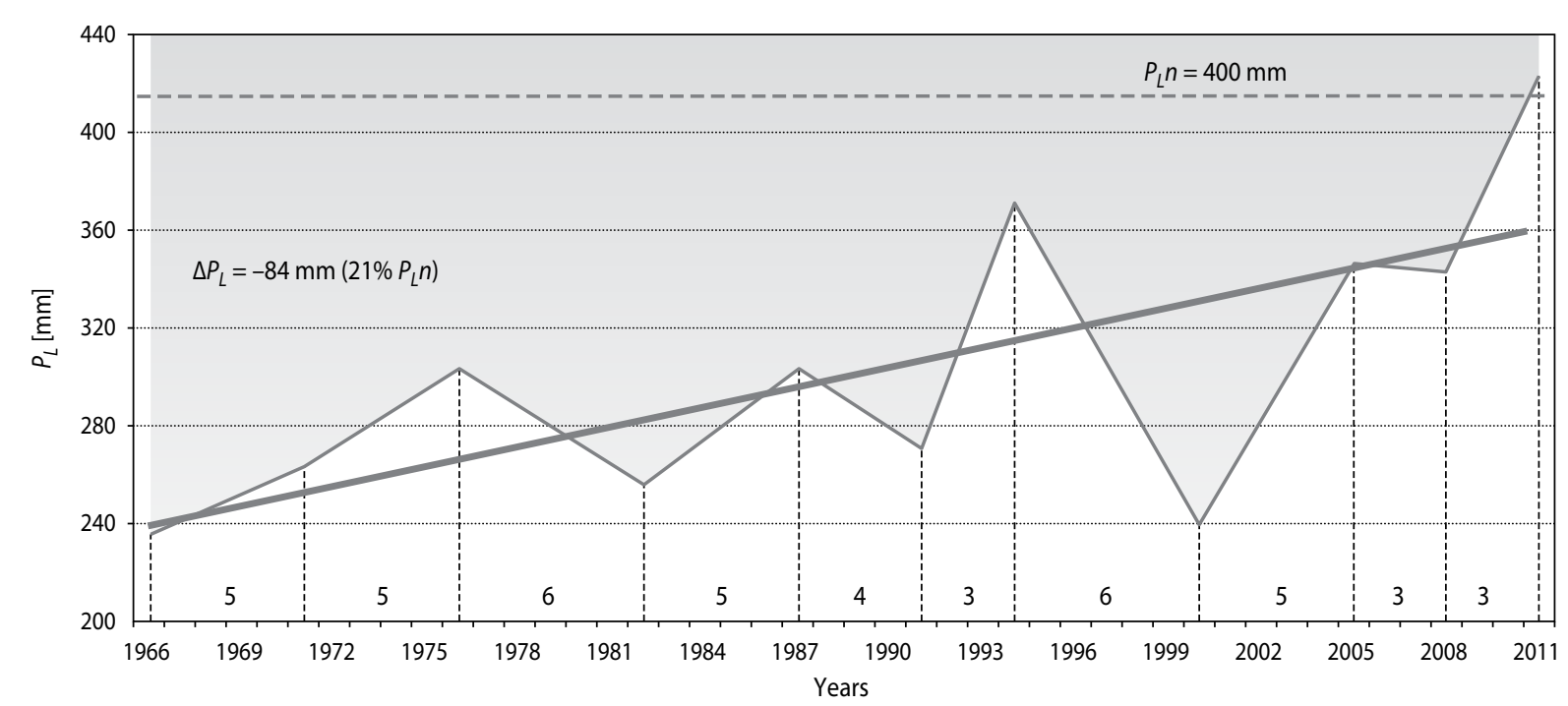

Fig. 6. Trend and cyclical nature of occurrence of minimum periodical rainfall in the summer 6-month period $\left(\mathrm{P}_{\mathrm{L}}\right)$ in the Łutownia catchment

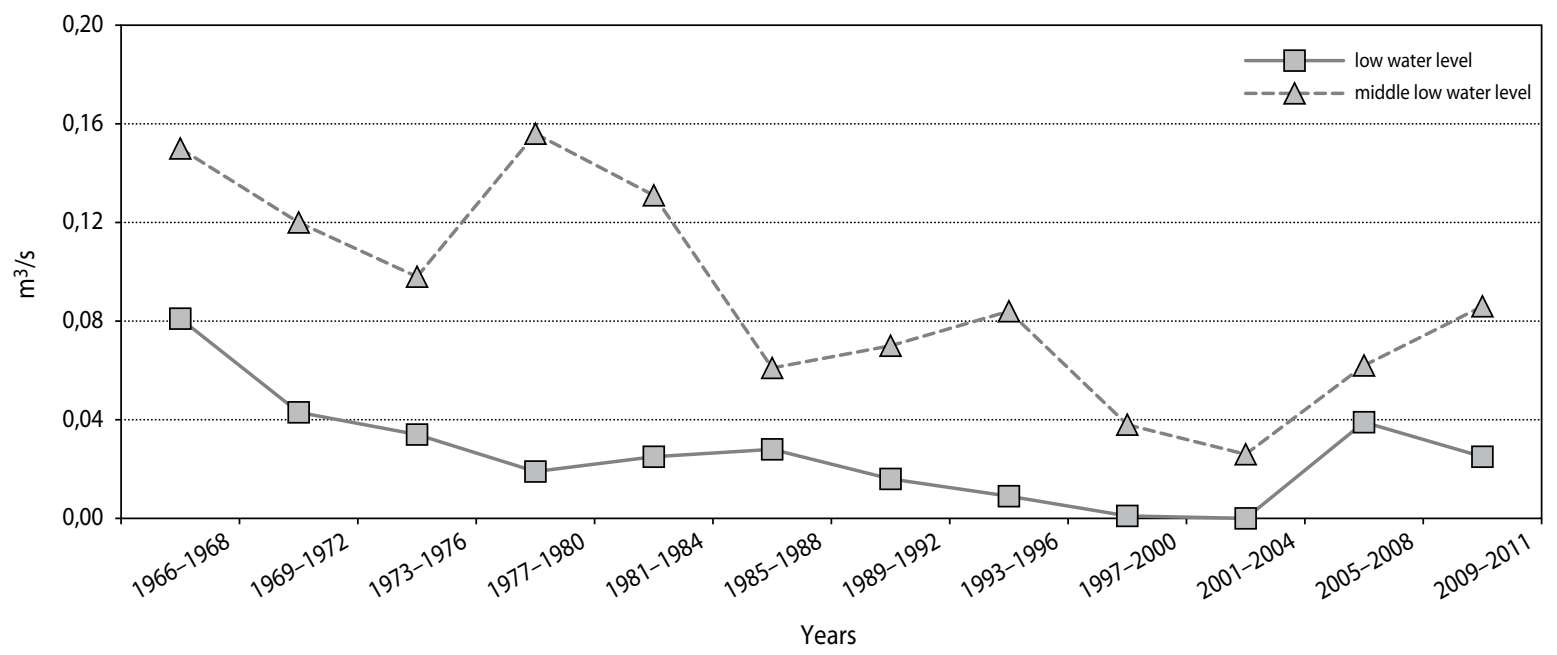

Fig. 7. Periodical discharges from the Łutownia river catchment, characteristic of the summer 6-month period (May-October)

Distribution of average monthly values (SNQ) and minimal (NNQ) discharges $\left(\mathrm{m}^{3} / \mathrm{s}\right)$ from the catchment of the Łutownia river were as follows:

\begin{tabular}{|l|c|c|}
\hline \multicolumn{1}{|c|}{ Month } & SNQ & NNQ \\
\hline 1 & 2 & 3 \\
\hline November & 0.15 & 0.02 \\
\hline December & 0.14 & 0.02 \\
\hline January & 0.17 & 0.02 \\
\hline February & 0.20 & 0.02 \\
\hline
\end{tabular}

\begin{tabular}{|l|c|c|}
\hline \multicolumn{1}{|c|}{1} & 2 & 3 \\
\hline March & 0.25 & 0.06 \\
\hline April & 0.26 & 0.02 \\
\hline May & 0.14 & 0.01 \\
\hline June & 0.10 & 001 \\
\hline July & 0.08 & 0.01 \\
\hline August & 0.08 & 0.00 \\
\hline September & 0.08 & 0.01 \\
\hline October & 0.12 & 0.02 \\
\hline
\end{tabular}


Minimal outflows occurred once in about 3 years in August, once in 4 years in September, once in 5 years in June and July, and once in about 9 years in May.

\section{Catchment retention capacity}

Forests have higher retention capacity when compared to that of agricultural lands. While discussing catchment retention capacity, there should be taken into account both the volume of precipitation water and duration of its maintenance in the soil profile, in ground and on the land surface including snow cover and vegetation. Good retention capacity of the primeval forest provides relatively stable conditions for nature expansion. In view of the process of retention capacity development, important roles are played by soil permeability and moisture as well its suction power (pF) (Król 1979; Maciaszek 1998). According to Maciaszek (1998) water capacity of forest soils ranges from $37.55 \%$ for acidic brown soils to $82.8 \%$ for bog soils. Retention of water in the forest soil profile of $1 \mathrm{~m}$ amounts from 106 to $204 \mathrm{~mm}$. In prevailing Poland's mineral and permeable soils water amounts are easy reinstated, however maintained shortly. Retention capacity in forest catchments with mineral soils depends not only on hydro-physical properties of the soil but also on the thickness of the zone of soil aeration (Suliński 1989; Tyszka 2008). After precipitation or snow melting, catchment recharge with groundwa- ter can sometimes last for a long time. Detailed investigation of water retention has to include recognition of all retention types including interception capacity of vegetation. In case of the Łutownia river catchment, we firstly determined overall approximate retention value as a result of the analysis of rainfall and summer runoff in subsequent decades (fig. 8). Relations rainfall-runoff were disturbed especially in 1970-2000 decades, i.e. during the period of changing conditions of the discharge process when rainfall-runoff relations were disturbed by durable retention. For example, large runoff was observed in the 80-ties, during the period of low rainfall $\left(\mathrm{P}_{\mathrm{L}}\right)$ and high air temperatures $\left(\mathrm{T}_{\mathrm{L}}\right)$.

Retention capacity $\left(\Delta \mathrm{R}_{1}\right)$ can be evaluated based on runoff changes in the summer 6 -month periods in the years with differentiated values of runoff coefficient $c=H_{L} / P_{L}$. Largest retention capacities were observed in the periods when small runoff occurred at high rainfall. At runoff $400 \mathrm{~mm}$ (close to normal), the difference between the years with edge values of runoff coeficient, i.e. hydrologically dry or wet, was $56 \mathrm{~mm}$. This value was $10 \mathrm{~mm}$ higher when evaluated for separate years (fig. 9).

Summer runoff is influenced not only by current rainfall, but also by the process of evapotranspiration and water recharge from melting snow. Fig.10 illustrates the relation between winter/summer rainfall $\left(\mathrm{P}_{\mathrm{Z}} / \mathrm{P}_{\mathrm{L}}\right)$ and

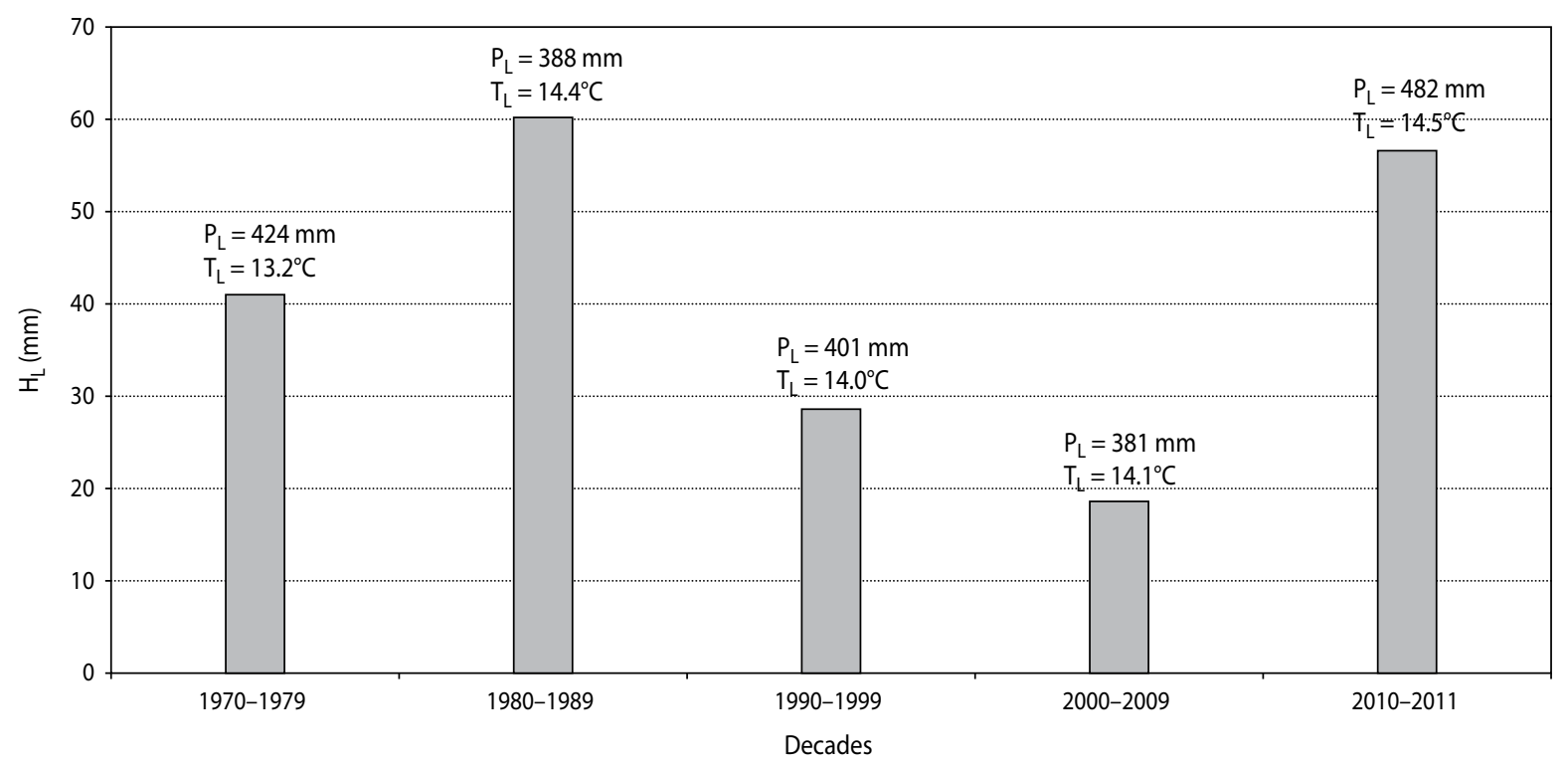

Fig. 8. Runoff in the summer and winter 6-month periods in 2010 and 2011 with reference to hydrothermal conditions 


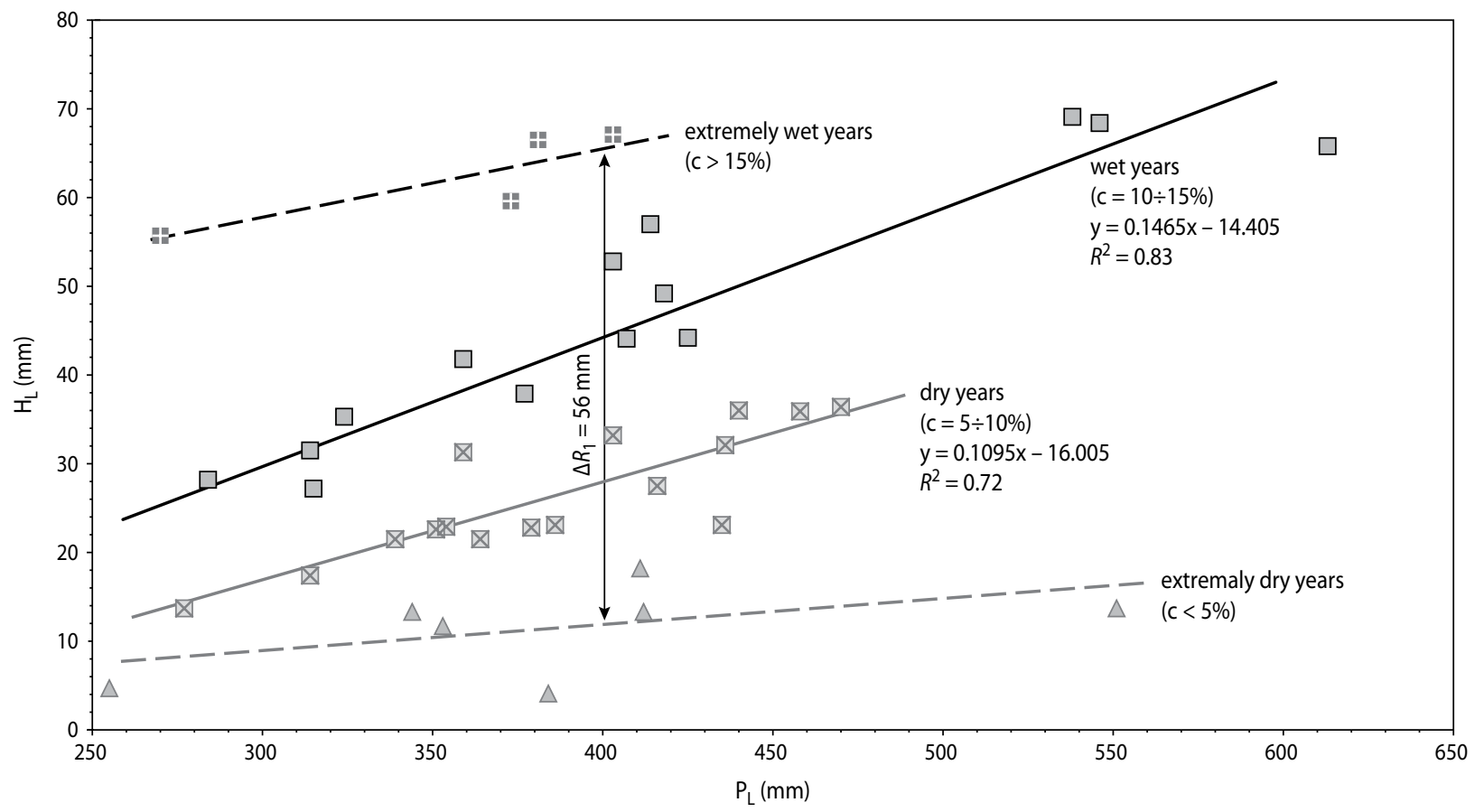

Fig. 9. Retention capacity $\left(\Delta \mathrm{R}_{1}\right)$ of forest habitats determined based on the difference of runoff in the years with edge values of runoff coefficient in the summer period $\mathrm{c}=\mathrm{H}_{\mathrm{L}} / \mathrm{P}_{\mathrm{L}}(\%)$

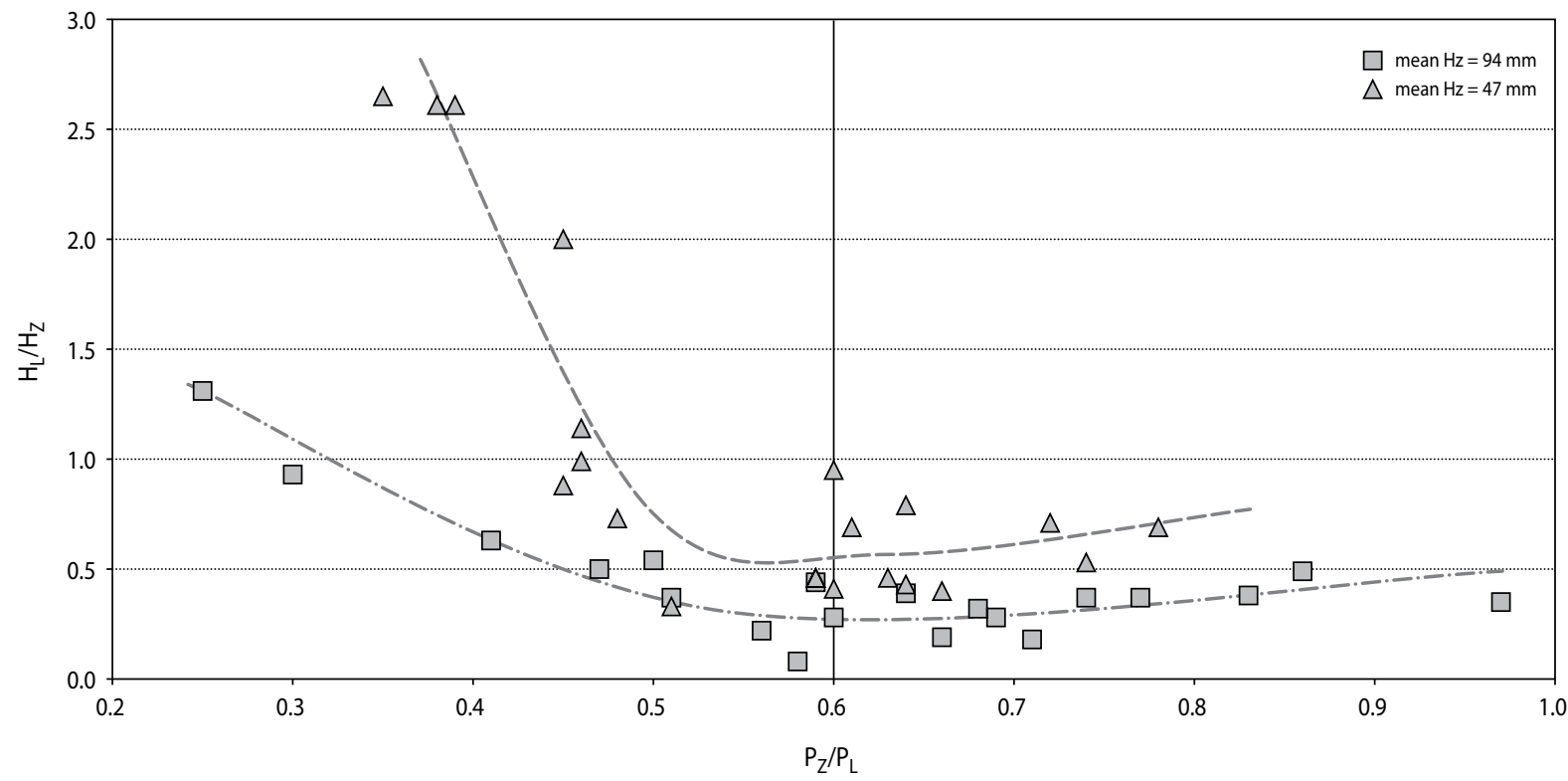

Fig. 10. Summer runoff as the effect of rainfall recharge in the summer and retention during the winter 6-month-period

summer/winter runoff $\left(\mathrm{H}_{\mathrm{L}} / \mathrm{H}_{\mathrm{Z}}\right)$ and shows that winter retention reserves are decisive of summer runoff if winter rainfall $\left(\mathrm{P}_{Z}\right)$ is higher than summer rainfall $\left(\mathrm{P}_{\mathrm{L}}\right)$ of about
$0.6 \mathrm{~mm}$. At equal values of rainfall in both 6-month periods, the increase of runoff can reach $20-30 \%$.

The above relation is best confirmed by the difference between winter and summer runoff $\left(\mathrm{H}_{\mathrm{Z}}-\mathrm{H}_{\mathrm{L}}\right)$ 


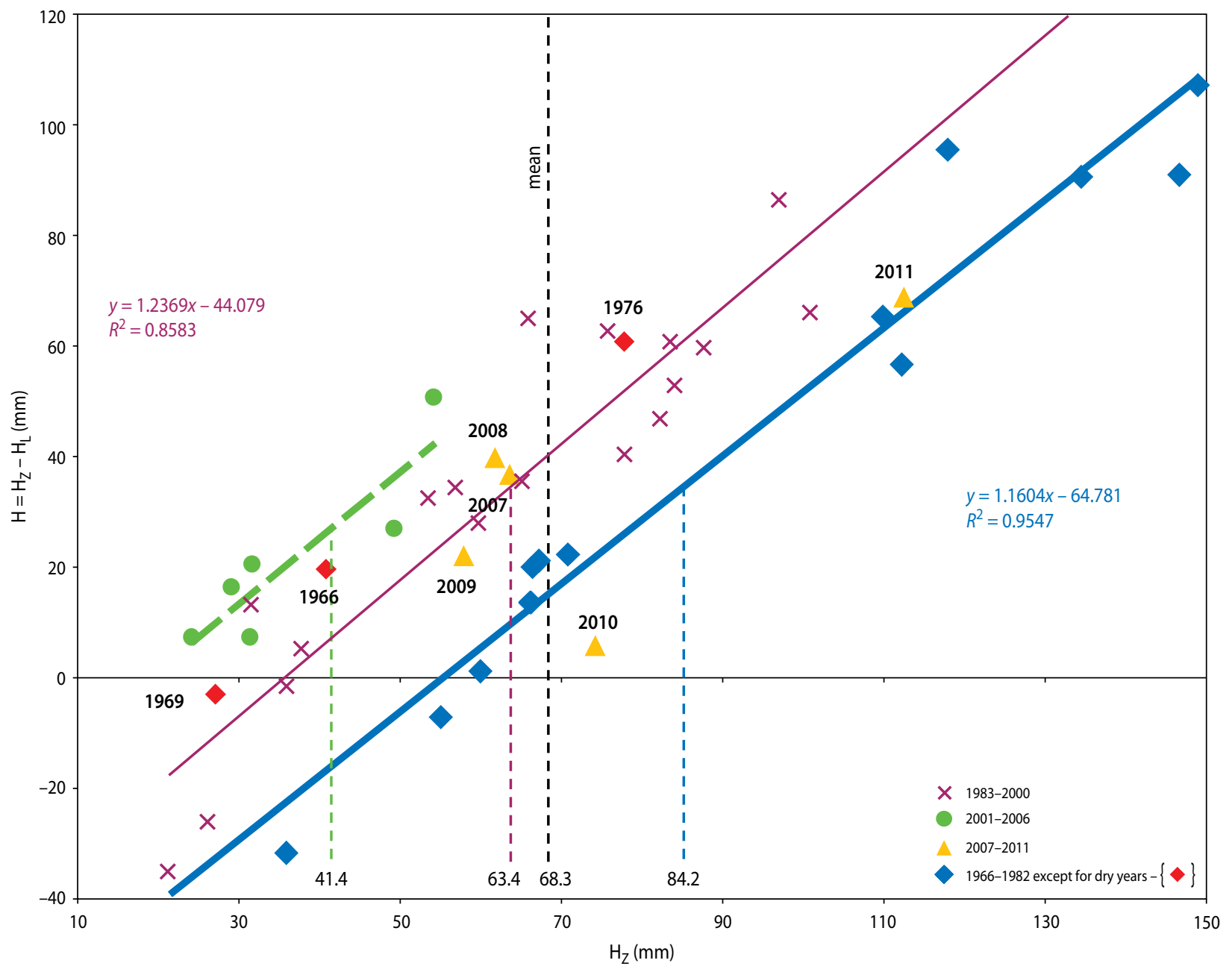

Fig. 11. Periodical changes of runoff in the winter 6-month period $\left(H_{Z}\right)$ as index value of catchment retention $\left(\Delta R_{2}=H_{Z}-H_{L}\right)$

which increases under the influence of increased winter runoff (Hz) (fig. 11). The difference can be treated as an index value of forecasted runoff in the summer 6-month period, which periodically changes in different phases of water resources fluctuations.

A decrease of winter runoff by $42.8 \mathrm{~mm}$ occurred between the beginning (1966-1982) and the end (2001-2006) of observations and the difference $\left(\Delta R_{2}\right)$ between winter and summer runoff was decreased by the similar quantity (fig. 11). Periodical character of retention changes was associated with hydrothermal conditions (fig. 12). There can be distinguished the periods with regular dependencies between summer runoff and air temperature:

- warm and wet 1970-ties, when runoff was decreasing with rising air temperature,
- cool 1980-ties with surplus water balance and characteristic of increasing with temperature runoff,

- summers, with close to normal temperatures ranging from $13.2-14.6^{\circ} \mathrm{C}$ and summer runoff $\left(\mathrm{H}_{\mathrm{L}}\right)$ 11-42 $\mathrm{mm}$ which was increasing with temperature,

- dry summers after 2000 with very low runoff which was increasing in summers with higher temperatures,

- wet summers after 2000 when runoff volumes and temperatures were similar to those observed at the beginning of investigation.

The results indicate that changes in hydrothermal conditions are of periodical character, and their recognition allows forecasting approximate summer discharge based on known volume of winter discharge. 


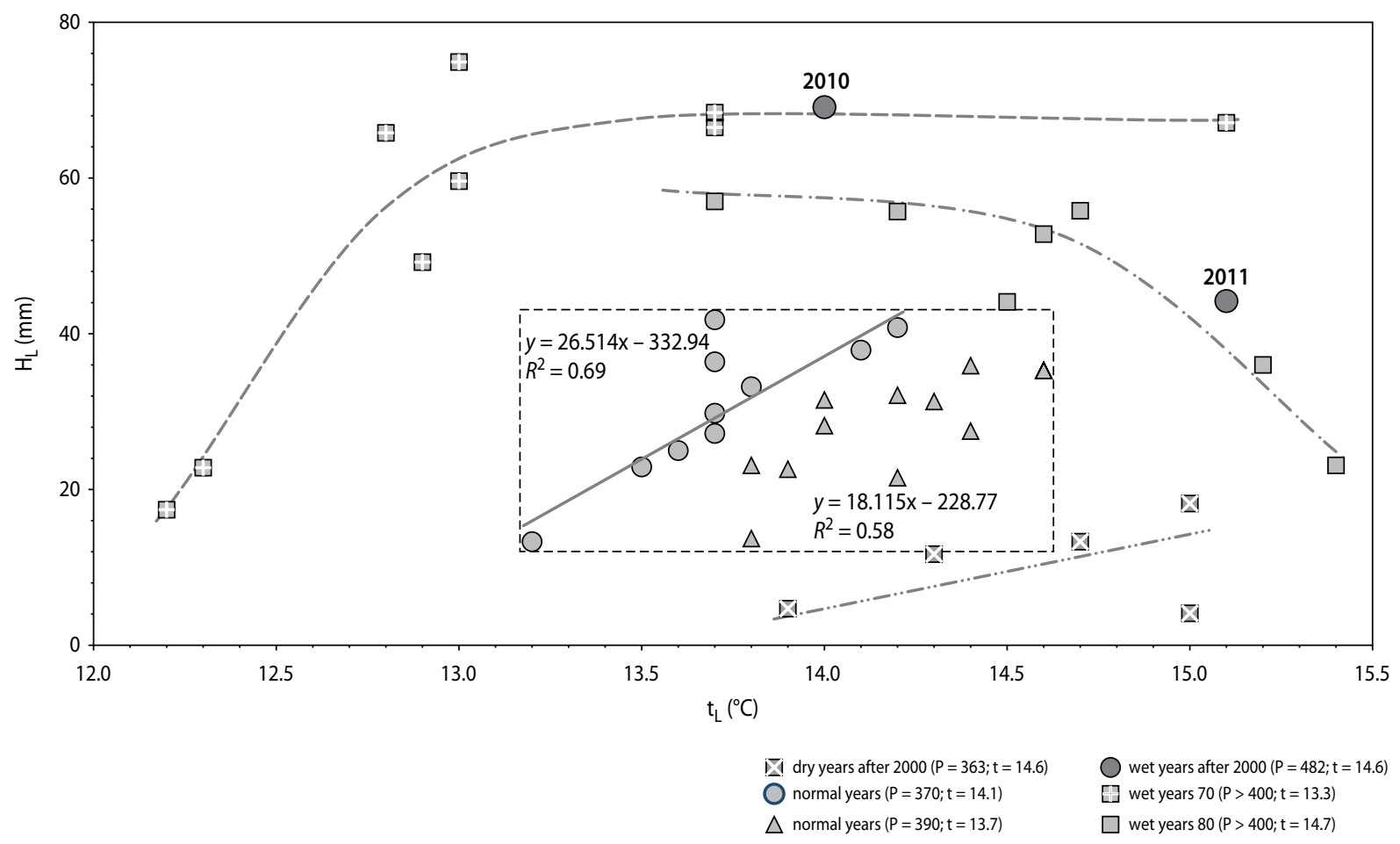

Fig. 12. Effects of temperature on runoff in the summer 6-month period in differentiated hydrothermal conditions

Two periods of time: 1983-2000 and 2001-2006 with similar rainfall and temperatures were chosen for the analyses in terms of finding other than climatic reasons of the decrease of retention resources. The difference between winter and summer runoff in the periods investigated was $17 \mathrm{~mm}$. This can be attributed to increased water needs of forest as a result of growing volume of large timber in stands.

\section{Discussion}

Determination of the role of forests in the catchment water cycle has become an issue focusing the attention of hydrologists as well as foresters, and has not yet been fully understood. External conditions which shape forest-water relations are so much environmentally complicated that actually each forest complex has to be approached separately with taking into account its hydro-climatic conditions as well as features of natural environment. The results of broad research undertaken in a range of geographical conditions, forest habitats and catchment areas of differentiated physi- ographic characteristics often concluded with opposite results. Divergent results on hydrological functions of forests were obtained by several researchers with recognized standing and professional experience (Dębski 1951; Lull, Sopper 1965; Bac 1968; Rachmanow 1970; Baumgartner 1971; Calder 2007). The direct changes in climatic conditions for forest functioning have recently become an additional concern in understanding relations between runoff and forest management within the catchment area. The results of attempts towards statistical interpretation of these relations are most often applicable only at a local level and are burdened with a broad range of measurement data which have to be constantly registered.

In the present study we attempted to evaluate water retention status based on the changes observed within the area of unique natural forest habitats of the Białowieża Primeval Forest. We used a narrow range of climatic and hydrological parameters however these were registered as unified measurement series during the long-term period. The data obtained served for interpretation of the reasons of observed changes as well as evaluation of water retention capacity - treated as the 
main feature of hydro-physical properties specific for forest ecosystems. The study was carried out with the aim to determine extreme changes in water resources, the knowledge of which can be used in the protection of nature valuable ecosystems. There were taken into account hydrological processes resulting from weather fluctuations and directional climatic trends, which are important in view of catchment runoff treated as a measure of territorial changes in water resources.

The results showed that the observed trend of decreasing water resources was accompanied by the increase of air temperature growing by $0.2^{\circ} \mathrm{C}$ every 10 years since the 1970-ties. Ongoing changes are indicated by among others decreasing Sielianinow's hydrothermal index, the value of which was about 1.8 in the 1970-ties and 1.45 in the years 2000-2009. The latter is associated with storm rainfalls and long-term droughts (Malzahn and Chomutowska 2009). The decrease of hydrothermal index (measured by the difference between rainfall and water runoff) results in water balance losses in the summer 6-month period (fig. 13) (Malzahn et al. 2012).

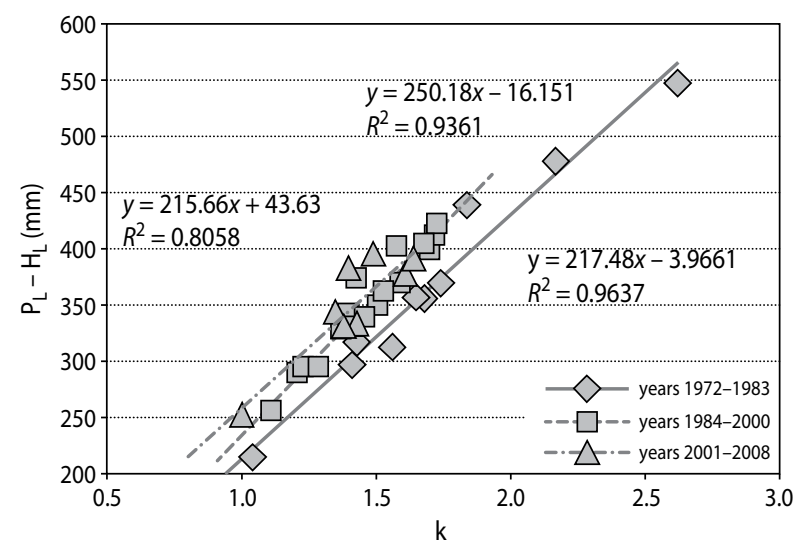

Fig. 13. Periodical differentiation of water balance losses during the summer 6-month period $\left(\mathrm{P}_{\mathrm{L}}-\mathrm{H}_{\mathrm{L}}\right)$ within the Łutownia river catchment according as Sielianinow's hydrothermal index

Besides current precipitation, the process of shaping discharge is influenced by the factors associated with water retention in forest habitats and fulfilling water needs of forest vegetation. The type of habitats and the intensity of tree biomass production affects directly evapotranspiration - the constituent of water balance (Suliński 1993; Kędziora and Ryszkowski 1998). Evap- otranspiration increases with rising temperature and precipitation. In the Białowieża Primeval Forest, during the research period, rainfall increased by $1.34 \mathrm{~mm} /$ year. This is a result of both moisture deficiency and needs for water in increasing tree biomass following the improvement of habitat fertility, decrease of soil pollution and limitations of logging. The changes of hydrological parameters of the Łutownia river catchment which is situated in the central part of the Białowieża Primeval Forest indicate that after 1983 there was shown a tendency to decrease water resources leading to limitation of possibilities to meet water needs of tree stands. Such phenomenon was also observed in many other forest complexes in Poland (fig. 14), however rainfalls in 2010 impeded its further progress.

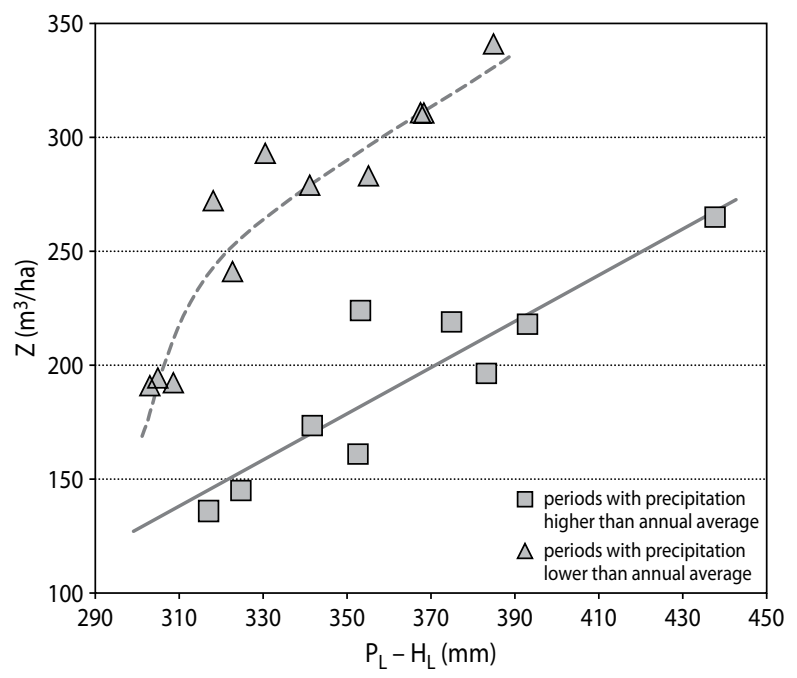

Fig. 14. Changes of water balance losses $(\mathrm{P}-\mathrm{H})$ in the summer 6-month period as a result of growth of stand volume $(Z)$ in lowland forest catchments (Tyszka 2007)

The occurrence of serious hydrological droughts within the area of the Białowieża Primeval Forest is a warning sign signalling that prevention activities should be undertaken towards avoiding degradation of valuable wetland habitats. In view of deficiency in precipitation during the summer 6-month period, we cannot anticipate overall improvement of water conditions in the whole Primeval Forest. Countermeasure activities should be focused on slow changes in moisture of the most sensitive habitats. However, water enrichment in one habitat generally leads to water depletion in the 
other one. Inadvertent activities of beavers, especially after the year 1995, are leading to increased water retention in river valleys, and on the other hand - to destabilization of water conditions within the areas located downstream. If the trend of dry years is maintained, there will have to be taken into consideration changes in stand species composition towards stands with lower water demands followed by possible extinction of some valuable flora and fauna species.

The results on relations between rainfall and runoff obtained in this study confirmed the view of Dębski (1951) that in Middle European Plain climatic conditions, forests play differentiated functions in the catchment water cycle depending on wet, dry or normal years. In the Białowieża Primeval Forest normal annual precipitation is $630 \mathrm{~mm}$. Precipitation in the summer 6-month period $350 \mathrm{~mm}$ leads to the increase of water use by forest stands, supplementation of water retention in soil and litter as well as water storage inside organisms and on the surface of plants. So as to assure stable water supply for forest stands under climatic conditions of the Middle European Plain, the increase of temperature by $1^{\circ} \mathrm{C}$ should be compensated by $14 \%$ increase of precipitation (Tyszka, Żakowicz 1998).

During last decades the above condition has not been fulfilled, thus the increase of balance losses has been observed. Suliński (1989) carried out a 3-year study on the effect of forest stands on catchment water properties within the highland catchment of the river Traczówka comprising pine stands. Runoff of $63 \%$ in the summer 6-month period explained ground water changes observed. In the years of observations, registered summer runoff differed by $61 \mathrm{~mm}$ which was a result of rainfall changes. Discharge volume was influenced by stand age which affected the depth of groundwater retention. Thus, the pine stand regulated retention capacity of the catchment investigated. The intensity of tree transpiration, changing with age, can also have additional influence on discharge volume (Boczoń 2004).

More radical methods of research on hydrological effects of forests, including evaluation of consequences of forest removal were applied in dissimilar nature conditions in the U.S.A. (Swank and Crossley 1988). The results indicated an increase of runoff and maximum flows, followed by deterioration of soil retention properties and changes in evaporation conditions.
According to the results obtained by Okoński and Miler (2010), retention properties of a catchment depend to a big extent on tree stand characteristics. These are determined by the effective precipitation, i.e. that part of precipitation which decides about the volume of rising waters. This could be important contribution to SCS-CN method which is used for hydrological forecasting in agricultural catchments. The overall retention status analyzed every six months can give a basis for the interpretation of differentiated effects on water rising caused by intensive precipitation or snow melting.

All the results on hydrological functions of forest give insight to the issue of the effects of forests on shaping the water cycle, however due to limited range of representativeness they have not so far been commonly applied in practice. Determination of retention capacity of forest is important both for sustainability of forest ecosystems and enchancement of the role of forests in regulating river discharge.

\section{Conclusions}

- Decreased river discharge volumes as well as the disturbed process of shaping the annual runoff cycle indicate deterioration of appropriate water conditions for functioning ecosystems of the Białowieża Primeval Forest. The minimal discharges are distinctive in terms of substantial volume decrease and earlier occurrence in the annual cycle.

- The decreasing trend of river runoff within the area of the Białowieża Primeval Forest observed in the years 1983-2009 was foremost a result of low rainfall during this period of time. Current decrease of river runoff should be also interpreted as a consequence of increased air temperatures, increased water needs of stands in changing habitat conditions as well as lately established small retention objects.

- Retention capacity of the catchment can be evaluated based on the differences between runoff in winter and summer 6-month periods or else between runoff in dry and wet years. During periodical drought in vegetation months, precipitation deficiency can be substituted to a big extent by after-winter retention resources. 
- The maximum water retention capacity of the habitats within the Białowieża Primeval Forest amounts for about $100 \mathrm{~mm}$ when assessed based on runoff differentiation in 6-month periods (excluding changes in water use by tree stands).

- Runoff from the catchment serves as a good measure of the status and changeability of the conditions needed for proper functioning of forest habitats. Feasibility and precision of measurements allow using the results on the changes observed for evaluating water retention status as well as short-term forecasting catchment water conditions. Enhancement of water cycle monitoring in forest catchments is in line with UE's law recommendations.

\section{References}

Bac S. 1968. Rola lasu w bilansie wodnym Polski. Folia Forestalia Polonica, Series A Forestry, 14, 5-65.

Baumgartner A. 1971. Einfluss energetischer Faktoren auf Klima. Produktion und Wasserumsatz in bewaldeten Einzugsgebieten. $15^{\text {th }}$ IUFRO- Congress in Gainsville (Flo), USA.

Boczoń A.2004. Zużycie wody przez dęby. Monografie Komitetu Gospodarki Wodnej PAN, 23, 123.

Byczkowski A. 1999. Hydrologia. Wydawnictwo SGGW, Warszawa.

Byczkowski A., Mandes B. 1998. Influence of catchment forested area on selected flows according to the regression analysis of those flows versus flow generation factors, case study for the north-east Poland. International Scientific Conference „Forest and Water" Cracow, 25-29 May, 107-114.

Calder J.R. 2007. Forest and water-ensuring forest benefits outweigh water costs. Forest Ecology and Management, 251, 110-120.

Ciepielowski A., Gutry-Korycka M., Tyszka J. 1992. Wykorzystanie cech lasu do opisu matematycznego odpływu ze zlewni. Prace Instytutu Badawczego Leśnictwa „Gospodarka wodna w lasach”, 155-164.

Dębski K. 1951. Wpływ lasu na stosunki hydrologiczne. Wiadomości Służby Hydrologicznej i Meteorologicznej, 3/4, 57-70.

Kędziora A., Ryszkowski L. 1998. Modification of evapotranspiration by different plant patches. Inter- national Scientific Conference „Forest and Water” Cracow, 25-29 May, 149-164

Król H. 1979. Zdolności retencyjne gleb różnych siedlisk leśnych. Sylwan, 1, 23-30.

Kucharska K., Tyszka J., Ciepielowski A. 1984. Rola lasów w wyrównaniu odpływu z małych zlewni w północno-wschodniej Polsce. Gospodarka Wod$n a, 4,107-110$.

Lull H.W., Sopper W.E. 1965. Prediction of overage annual and seasonal streamflow of physiographic units in the northeast. International symposium on forest hydrology, Pennsylvania.

Maciaszek W. 1998. Soil as a natural water reservoir. International Scientific Conference „Forest and Water", Cracow, 25-29 May, 290-299.

Majer A. 2004. Zmiany w siedliskach hydrogenicznych w ocenie Biura Urządzania Lasu i Geodezji Leśnej. Seminarium „Zagrożenia leśnych siedlisk hydrogenicznych w Puszczy Białowieskiej”. Białowieża 21.05.2004.

Malzahn E., Chomutowska H. 2009. Ocena zmian czynników abiotycznych w Puszczy Białowieskiej. Zeszyty Problemowe Postępów Nauk Rolniczych, 540, 247-258.

Malzahn E., Pierzgalski E., Tyszka J., Janek M. 2012. Zmiany warunków klimatycznych i wodnych w środowisku lasów naturalnych Puszczy Białowieskiej. Sprawozdanie z realizacji badań - temat BLP-361, Biblioteka IBL.

Okoński B., Miler A.T. 2010. Adaptacja metody SCS-CN dla obliczania opadu efektywnego w zlewniach leśnych. Monografie Komitetu Inżynierii Środowiska PAN, vol. 68, tom 1 „Hydrologia w inżynierii i gospodarce wodnej” (ed.: B. Więzik), 143-152.

Olszewski J. 1986. Rola ekosystemów leśnych w modyfikacji klimatu lokalnego Puszczy Białowieskiej. Ossolineum, Wrocław.

Pasławski Z. 1973. Metody hydrometrii rzecznej IMGW. Instrukcje i podręczniki nr 115.

Pierzgalski E., Tyszka J., Boczoń A. 2002a. Zróżnicowanie odpływu w małych zlewniach rzecznych w rejonie Puszczy Białowieskiej. Prace Instytutu Geografii Akademii Świętokrzyskiej, 7, 133-145.

Pierzgalski E., Boczoń A., Tyszka J. 2002b. Zmienność opadów i położenia wód gruntowych w Białowieskim Parku Narodowym. Kosmos, 51 (4), 415-425. 
Pierzgalski E., Tyszka J. 2005. Water outflow during drought years in watersheds with various forest cover. Annals of Warsaw Agricultural University, 36, 21-28.

Pierzgalski E., Tyszka J., Stolarek A. 2006. Zmienność odpływu wody ze zlewni rzeki Łutowni (Puszcza Białowieska) w latach 1966-2000. Leśne Prace Badawcze, 1, 21-36.

Rachmanow W. 1970. Zavisimost recznogo stoka ot lesistosti bassiejnow. Moskwa.

Suliński J. 1989. Szczegółowy bilans wodny zlewni potoku Traczówka $\left(24 \mathrm{~km}^{2}\right)$ w Puszczy Niepołomickiej. Acta Agraria et Silvestria, Series Silvestris, 28, 105-123.

Suliński J. 1993. Modelowanie bilansu wodnego w wymianie miedzy atmosfera, drzewostanem i gruntem przy użyciu kryteriów ekologicznych. Zeszyty $\mathrm{Na}$ ukowe AR w Krakowie, 179, 1-10.
Swank W.T., Crossley Jr. D.A. 1988. Forest Hydrology and Ecology at Coweeta. Ecological Studies, vol. 66. Springer-Verlag New York. Inc.

Tyszka J., Żakowicz S. 1998. Changes in soil retention and geochemical properties of water in selected forest ecosystems of Northern Poland. International Scientific Conference „Forest and Water”, Cracow, 25-29 May, 198-208.

Tyszka J. 2007. Kształtowanie się zasobów wodnych lasu w zmieniających się warunkach klimatycznych. In: Quo vadis, forestry? (ed.: Z. Sierota). Instytut Badawczy Leśnictwa, Sękocin Stary, 440-448.

Tyszka J. 2008. Hydrologiczne funkcje lasu w małych zlewniach rzecznych. Prace IBL. Rozprawy i Monografie, 10, 1-215. 